\title{
On High Order Strong Stability Preserving Runge-Kutta and Multi Step Time Discretizations
}

\author{
Sigal Gottlieb ${ }^{1}$
}

Received October 1, 2003; accepted (in revised form) March 15, 2004

\begin{abstract}
Strong stability preserving (SSP) high order time discretizations were developed for solution of semi-discrete method of lines approximations of hyperbolic partial differential equations. These high order time discretization methods preserve the strong stability properties-in any norm or seminorm - of the spatial discretization coupled with first order Euler time stepping. This paper describes the development of SSP methods and the recently developed theory which connects the timestep restriction on SSP methods with the theory of monotonicity and contractivity. Optimal explicit SSP Runge-Kutta methods for nonlinear problems and for linear problems as well as implicit Runge-Kutta methods and multi step methods will be collected.
\end{abstract}

KEY WORDS: Strong stability preserving; Runge-Kutta methods; multi step methods; high order accuracy; time discretization.

AMS (MOS) SUBJECT CLASSIFICATION: 65M20; 65L06.

\section{INTRODUCTION TO SSP METHODS}

In the use of numerical methods for approximating solutions of PDEs we typically rely on linear stability theory to guarantee convergence. The celebrated Lax equivalence theorem (see [34] Theorem 1.5.1) states that for a linear method consistent with a linear problem, stability is necessary and sufficient for convergence. Strang [33] extended this result, and showed that for nonlinear problems if an approximation is consistent and its linearized version is $L_{2}$ stable, then for sufficiently smooth problems this approximation is convergent. However, solutions of hyperbolic partial differential equations (PDEs) are frequently discontinuous. In this case,

\footnotetext{
${ }^{1}$ Department of Mathematics, University of Massachusetts Dartmouth, USA. E-mail: sg@cfm.brown.edu
} 
the conditions of Strang's theorem are not fulfilled, and linear stability theory no longer guarantees convergence. Consequently, a tremendous amount of effort has been placed on the development of high order spatial discretizations which, when coupled with the forward Euler time stepping method, have the desired nonlinear stability properties for approximating the discontinuous solutions of hyperbolic PDEs.

When solving time dependent PDEs such as the hyperbolic conservation law of the form $u_{t}+f(u)_{x}=0$, the spatial derivative $f(u)_{x}$ is discretized by a carefully chosen nonlinearly stable finite difference or finite element approximation, [e.g. 3,11, 18, 21, 23, 35, 36] to obtain a semidiscrete method of lines scheme. If the spatial discretization to $f(u)_{x}$ is denoted $-L(u)$, and we let $u$ represent the vector of values in space, the PDE above becomes an ordinary differential equation (ODE) system in time $u_{t}=L(u)$, which can be solved by an ODE solver. The discretization $L$ is chosen so that the stability properties of the spatial discretization are guaranteed when used with the first order forward Euler method as the ODE solver for a sufficiently small time step dictated by the CFL condition.

However, for actual computation higher order time discretizations are usually needed, and there is no guarantee that the nonlinearly stable spatial discretization would necessarily produce stable results when coupled with a linearly stable higher order time discretization. In fact, numerical evidence [7,8] shows that oscillations may occur when using a linearly stable, high-order method, which does not preserve the stability properties of forward Euler, even if the same spatial discretization is TVD when combined with the first-order forward Euler time-discretization. This is a compelling reason to develop and use time discretization methods which preserve the stability properties of forward Euler.

Strong stability preserving (SSP) time discretization methods were developed to address the need for nonlinear stability properties in the time discretization, as well as the spatial discretization, of hyperbolic PDEs. The idea behind SSP methods is to assume that the first order forward Euler time discretization of the method of lines ODE is strongly stable under a certain norm, when the time step $\Delta t$ is suitably restricted, and then try to find a higher order time discretization (Runge-Kutta or multi step) that maintains strong stability for the same norm, perhaps under a different time step restriction. The class of high order SSP time discretization methods for the semi-discrete method of lines approximations of PDEs was developed in [28, 29] and called TVD (Total Variation Diminishing) time discretizations. This class of methods was further studied in $[6,7,14,25-27,30,31]$. These methods preserve the stability properties of forward Euler in any norm or semi norm. In fact, since the stability 
arguments are based on convex decompositions of high-order methods in terms of the first-order Euler method, any convex function (such as the cell entropy stability property of high order schemes studied in [22, 24] will be preserved by SSP high-order time discretizations.

Over the last few years, increasingly sophisticated mathematical and numerical techniques have been used to develop new and optimal SSP methods. The goal of this paper is to describe the Shu-Osher SSP theory and the recent developments, both numerical and theoretical, in this field, and to collect the main results and the most useful, in terms of computational cost, SSP methods. The paper is organized as follows: The SSP theory and Shu-Osher representation of explicit Runge-Kutta methods is described in Sec. 2, as well as the results on order barriers and optimal methods for linear problems and nonlinear problems, and low storage RK methods. The results for explicit SSP multi step methods appear in Sec. 3, and for implicit SSP RK and multi step methods in Sec. 4. Finally, the theory linking the CFL coefficient for SSP methods and the radius of absolute monotonicity is described in Sec. 5. The study of SSP generalized linear methods (Runge-Kutta and multi step hybrids) which appear in [8] will not be reviewed here, as the resulting methods are less computationally efficient than either the Runge-Kutta or the multi step methods.

\section{EXPLICIT SSP RUNGE-KUTTA METHODS}

In this section we review the SSP theory for explicit Runge-Kutta methods which approximate the solution of the ODE

$$
u_{t}=L(u)
$$

which arises from the discretization of the spatial derivative in the PDE

$$
u_{t}+f(u)_{x}=0
$$

where the spatial discretization $L(u)$ is chosen so that

$$
u^{n+1}=u^{n}+\Delta t L\left(u^{n}\right),
$$

satisfies the strong stability requirement $\left\|u^{n+1}\right\| \leqslant\left\|u^{n}\right\|$ in some norm $\|\cdot\|$, under the CFL condition

$$
\Delta t \leqslant \Delta t_{F E} .
$$


In [29], a general $m$ stage Runge-Kutta (RK) method for is written in the form:

$$
\begin{aligned}
u^{(0)} & =u^{n}, \\
u^{(i)} & =\sum_{k=0}^{i-1}\left(\alpha_{i, k} u^{(k)}+\Delta t \beta_{i, k} L\left(u^{(k)}\right)\right), \quad \alpha_{i, k} \geqslant 0, \quad i=1, \ldots, m, \\
u^{n+1} & =u^{(m)} .
\end{aligned}
$$

Consistency requires that $\sum_{k=0}^{i-1} \alpha_{i, k}=1$.

When $\beta_{i, k}$ is negative, $\beta_{i, k} L\left(u^{(k)}\right)$ is replaced by $\beta_{i, k} \tilde{L}\left(u^{(k)}\right)$, where $\tilde{L}$ approximates the same spatial derivative(s) as $L$, but the strong stability property $\left\|u^{n+1}\right\| \leqslant\left\|u^{n}\right\|$, holds for the first order Euler scheme, solved backward in time, i.e.,

$$
u^{n+1}=u^{n}-\Delta t \tilde{L}\left(u^{n}\right)
$$

This can be achieved, for hyperbolic conservation laws, by solving the negative in time version of (2.2),

$$
u_{t}-f(u)_{x}=0 .
$$

Numerically, the only difference is the change of upwind direction. Clearly, $\tilde{L}$ can be computed with the same cost as that of computing $L$. Thus, if $\alpha_{i, k} \geqslant 0$, all the intermediate stages in (2.5), $u^{(i)}$, are simply convex combinations of backward in time Euler and forward Euler operators, with $\Delta t$ replaced by $\left|\beta_{i, k}\right| / \alpha_{i, k} \Delta t$. Therefore, any norm, semi-norm or convex function property satisfied by the backward in time and forward in time Euler methods will be preserved by the RK method.

Theorem 2.1. ([29] Section 2). If the forward Euler method combined with the spatial discretization $L$ in (2.3) is strongly stable under the CFL restriction (2.4), i.e.

$$
\left\|u^{n}+\Delta t L\left(u^{n}\right)\right\| \leqslant\left\|u^{n}\right\|,
$$

and if Euler's method solved backward in time in combination with the spatial discretization $\tilde{L}$ in (2.6) is also strongly stable under the CFL restriction (2.4), i.e.

$$
\left\|u^{n}-\Delta t \tilde{L}\left(u^{n}\right)\right\| \leqslant\left\|u^{n}\right\|,
$$

then the RK method (2.5) is SSP $\left\|u^{n+1}\right\| \leqslant\left\|u^{n}\right\|$, under the CFL restriction, 


$$
\Delta t \leqslant c \Delta t_{F E}, \quad c=\min _{i, k} \frac{\alpha_{i, k}}{\left|\beta_{i, k}\right|},
$$

provided $\beta_{i, k} L$ is replaced by $\beta_{i, k} \tilde{L}$ whenever $\beta_{i, k}$ is negative.

The research in the field of SSP methods centers around the search for high order SSP methods where the CFL coefficient $c$ in the timestep restriction (2.7) is as large as possible. Many optimal methods have been found for the class of problems, where all the $\beta_{i, k} \mathrm{~s}$ are nonnegative. These methods include the case where there are more stages than required for the order, in order to maximize the CFL coefficient. Although the additional stages increase the computational cost, this is usually more than offset by the larger stepsize that may be taken.

It would seem that if both $L\left(u^{(k)}\right)$ and $\tilde{L}\left(u^{(k)}\right)$ must be computed for the same $k$, the computational cost as well as storage requirement for this $k$ is doubled. For this reason, negative $\beta_{i, k}$ were avoided whenever possible in [6-8, 25, 32]. However, since, as shown in Proposition 3.3 of [7] and Theorem 4.1 in [25], it is not always possible to avoid negative $\beta_{i, k}$, recent studies (e.g. [10, 26, 27]) have considered efficient ways of implementing negative $\beta_{i, k}$. First, inclusion of negative $\beta_{i, k}$, even when not absolutely necessary, may raise the CFL coefficient enough to compensate for the additional computational cost incurred by $\tilde{L}$. Second, since $\tilde{L}$ is, numerically, the downwind version of $L$, it is possible to compute both $L$ and $\tilde{L}$ without doubling the computational cost. For example, for the fifth order weighted ENO scheme, computing both $L$ and $\tilde{L}$ for a scalar conservation law using an efficient algorithm will increase the number of floating point operations by only $29 \%$ over the cost of computing only $L[10]$. Finally, if $L$ and $\tilde{L}$ do not appear for the same $k$, then neither the computational cost nor the storage requirement is increased.

\subsection{Optimal SSP Runge-Kutta Methods for Nonlinear Problems}

Since SSP methods were developed for use with hyperbolic conservation laws, most of the research to date has been in the derivation of SSP methods for nonlinear spatial discretizations. This is necessary because high order stable schemes for hyperbolic PDEs with discontinuous solutions are nonlinear even when the underlying PDE is linear.

In Sec. 2 of [29], optimal explicit SSPRK schemes up to third order were found with CFL coefficient $c=1$. In the following, $(\mathrm{m}, \mathrm{p})$ denotes an $m$-stage $p$ th order method: 
SSPRK (2, 2): If we require $\beta_{i, k} \geqslant 0$, then an optimal second order SSP RK method (2.5) is given by

$$
\begin{aligned}
u^{(1)} & =u^{n}+\Delta t L\left(u^{n}\right) \\
u^{n+1} & =\frac{1}{2} u^{n}+\frac{1}{2} u^{(1)}+\frac{1}{2} \Delta t L\left(u^{(1)}\right)
\end{aligned}
$$

with a CFL coefficient $c=1$ in (2.7).

SSPRK (3, 3): If we require $\beta_{i, k} \geqslant 0$, An optimal third order SSP RK method (2.5) is given by

$$
\begin{aligned}
u^{(1)} & =u^{n}+\Delta t L\left(u^{n}\right) \\
u^{(2)} & =\frac{3}{4} u^{n}+\frac{1}{4} u^{(1)}+\frac{1}{4} \Delta t L\left(u^{(1)}\right) \\
u^{n+1} & =\frac{1}{3} u^{n}+\frac{2}{3} u^{(2)}+\frac{2}{3} \Delta t L\left(u^{(2)}\right),
\end{aligned}
$$

with a CFL coefficient $c=1$ in (2.7).

Although both these methods have CFL coefficient $c=1$, which permits a time step of the same size as forward Euler would permit, it is clear that the computational cost is double and triple (respectively) that of the forward Euler. Thus, we find is useful to define the effective $C F L$ as $c_{\text {eff }}=$ $c / l$, where $l$ is the number of computations of $L$ and $\tilde{L}$ required per time step. In the case of $\operatorname{SSP}(2,2)$ and $\operatorname{SSP}(3,3)$ the effective CFL is $c_{\text {eff }}=1 / 2$ and $c_{\text {eff }}=1 / 3$, respectively. Of course, while in this case the increase in the order of the method makes this additional computational cost acceptable, the notion of the effective CFL is useful when comparing two methods of the same order.

$\operatorname{SSPRK}(3,3)$ is widely known as the Shu-Osher method, and is probably the most commonly used SSP RK method. Although this method is only third order accurate, it is most popular because of its simplicity, its classical linear stability properties, and because finding a fourth order SSP RK method proved difficult. In [7] (Proposition 3.3) it was proved that all four stage, fourth order RK methods with positive CFL coefficient $c$ in (2.7) must have at least one negative $\beta_{i, k}$. Thus, we must contend with the appearance of $\tilde{L}$ or additional stages. Spiteri and Ruuth ([31, 32]) developed fourth order methods with $m=5,6,7$ and 8 stages. The most popular fourth order method is the $m=5$ stage method with nonnegative $\beta_{i, k} \mathrm{~s}$ : 
SSPRK(5,4): ([27] Table 4.3, [17, 31]) The 5 stage fourth order SSPRK developed by Ruuth and Spiteri

$$
\begin{aligned}
u^{(1)}= & u^{n}+0.391752226571890 \Delta t L\left(u^{n}\right) \\
u^{(2)}= & 0.444370493651235 u^{n}+0.555629506348765 u^{(1)} \\
& +0.368410593050371 \Delta t L\left(u^{(1)}\right) \\
u^{(3)}= & 0.620101851488403 u^{n}+0.379898148511597 u^{(2)} \\
& +0.251891774271694 \Delta t L\left(u^{(2)}\right) \\
u^{(4)}= & 0.178079954393132 u^{n}+0.821920045606868 u^{(3)} \\
& 0.544974750228521 \Delta t L\left(u^{(3)}\right), \\
u^{n+1}= & 0.517231671970585 u^{(2)} \\
& +0.096059710526147 u^{(3)}+0.063692468666290 \Delta t L\left(u^{(3)}\right) . \\
& +0.386708617503269 u^{(4)}+0.226007483236906 \Delta t L\left(u^{(4)}\right)
\end{aligned}
$$

is SSP with CFL coefficient $c=1.508$, and effective CFL $c_{\text {eff }}=0.377$, which means that this method is more efficient, as well as higher order, than the popular $\operatorname{SSP}(3,3)$. In Sec. 3.2 of [27] the optimality of this scheme was guaranteed using an approach based on global optimization.

Ruuth and Spiteri ([25] Proposition 4.1) also proved that any SSPRK with nonzero CFL of order $p>4$ will have negative $\beta_{i, k}$. It therefore becomes necessary to include $\tilde{L}$ in any method of order 5 or above. Ruuth and Spiteri explore efficient fifth order schemes in [26]. These methods will probably become increasingly popular as the need for higher order methods arises, and as ways of computing $\tilde{L}$ more efficiently are found.

\subsection{Low Storage Methods for Nonlinear Problems}

Storage is usually an important consideration for large scale scientific computing in three space dimensions. Therefore low storage RK methods $([1,15,37])$, which only require 2 or 3 storage units per ODE variable, may be desirable. In [7, 8, 26, 27], some SSP low storage RK methods were studied. In [27], Ruuth presents many low storage schemes resulting from intensive global optimization routines. Some of these methods are guaranteed optimal, others are the best found in extensive numerical searches. Ruuth considered Williamson schemes [37] which require two units of storage per step and Van Der Houwen and Wray (described in [15]) schemes requiring two or three registers of storage. Negative $\beta_{i, k} \mathrm{~s}$ are allowed in these methods, as long as all the negative coefficients are associated with the same superscript, so that for each $U^{k}$ either $L$ or $\tilde{L}$ appears, 
but not both, and so the low storage property is not destroyed. The interested reader is referred to [27], where ten low storage methods, of order $p=3$ and $p=4$ and stages $m=3,4,5$ are given. One such very useful method is:

Method LS(5, 3) ([27] Sec. 4.) The $m=5, p=3$ Williamson low storage method

$$
\begin{aligned}
U^{(0)} & =u^{n}, \\
d U^{(1)} & =\Delta t L\left(U^{(0)}\right), \\
U^{(1)} & =U^{(0)}+0.713497331193829 d U^{(0)}, \\
d U^{(2)} & =-4.344339134485095 d U^{(1)}+\Delta t L\left(U^{(1)}\right), \\
U^{(2)} & =U^{(1)}+0.133505249805329 d U^{(1)}, \\
d U^{(3)} & =\Delta t L\left(U^{(2)}\right), \\
U^{(3)} & =U^{(2)}+0.713497331193929 d U^{(2)}, \\
d U^{(4)} & =-3.770024161386381 d U^{(3)}+\Delta t L\left(U^{(3)}\right), \\
U^{(4)} & =U^{(3)}+0.149579395628565 d U^{(3)}, \\
d U^{(5)} & =-3.046347284573284 d U^{(4)}+\Delta t L\left(U^{(4)}\right), \\
U^{(5)} & =U^{(4)}+0.384471116121269 d U^{(4)}, \\
u^{n+1} & =U^{(5)}
\end{aligned}
$$

is numerically optimal, with CFL coefficient $c=1.4$ and no $\tilde{L}$ computations. This method may be used instead of $\operatorname{SSP}(3,3)$ with almost the same computational cost: $\operatorname{SSP}(3,3)$ has $c_{\text {eff }}=1 / 3$ and the low storage method $\operatorname{LS}(5,3)$ has $c_{\text {eff }}=0.28$. This increase in cost is reasonable when storage is a critical need.

\subsection{Optimal Methods for Linear Constant Coefficient Problems}

Although SSP methods were created to provide nonlinearly stable time discretizations for nonlinearly stable spatial discretizations of hyperbolic PDEs, they have proven useful for linear problems as well. In [20], the authors used the energy method to analyze the stability of RK methods for ODEs resulting from coercive approximations such as those in [9]. Using this method it can be proven, for example, that the fourth order RK method preserved the desired stability property with a CFL number of $c=1 / 31$. However, when this method was analyzed using the SSP ideas, it became clear that the CFL number was, in fact $c=1$. Thus, linear SSP RK methods became useful from the point of view of stability analysis. Once the class of linear SSP RK methods was developed, it gained popu- 
larity because of the guarantee of provable stability. This section describes some of the main results in this field:

Consider the ODE (2.1), where $L$ is a linear constant coefficient operator which can be written as a finite dimensional matrix, so that we denote $L(u)=L u$. In this case SSP RK methods may be found with higher CFL coefficients than in the nonlinear case or the linear variable coefficient case $L(t, u)=L(t) u$. If we increase the number of stages $m$ without increasing the order $p$, we obtain SSP RK methods with higher CFL coefficients. In [25] and in [6], it was shown that the family of $m$-stage, $p$ th order SSP RK methods (2.5) with nonnegative coefficients $\alpha_{i, k}$ and $\beta_{i, k}$ has CFL coefficient $c$ at most $c=m-p+1$. However, this CFL coefficient is only a barrier, and is not generally obtainable. In Table I we quote Kraaijevanger's results [16], which were used by Higueras ([12] Table IV) for determining the optimal CFL coefficient of a $m$ stage, linear $p$ th order SSP RK method; furthermore, we include the corresponding effective CFL. These results were derived, as will be explained in Sec. 5, using the connections between contractivity theory and the radius of absolute monotonicity. Of course, bounds on the linear case apply also to the nonlinear case, which is far more restrictive.

SSP RK methods which obtain these barriers were considered in [6, $8,25]$. We list the results for SSP RK methods for linear constant coefficient problems:

SSPRK linear (m, m): ([8] Proposition 3.2) The class of $m$ stage schemes given by:

$$
\begin{aligned}
u^{(i)} & =u^{(i-1)}+\Delta t L u^{(i-1)}, \quad i=1, \ldots, m-1, \\
u^{(m)} & =\sum_{k=0}^{m-2} \alpha_{m, k} u^{(k)}+\alpha_{m, m-1}\left(u^{(m-1)}+\Delta t L u^{(m-1)}\right),
\end{aligned}
$$

where $\alpha_{1,0}=1$ and

$$
\begin{aligned}
\alpha_{m, k} & =\frac{1}{k} \alpha_{m-1, k-1}, \quad k=1, \ldots, m-2, \\
\alpha_{m, m-1} & =\frac{1}{m !}, \quad \alpha_{m, 0}=1-\sum_{k=1}^{m-1} \alpha_{m, k}
\end{aligned}
$$

is an $m$-order linear RK method which is SSP with CFL coefficient $c=1$, which is optimal among all $m$ stage, $p=m$ order SSPRK methods with nonnegative coefficients. The effective CFL is $c_{\text {eff }}=1 / \mathrm{m}$. Table II includes the coefficients of these methods up to order 8 . 
Table I. Optimal CFL Coefficients $c$, and the Corresponding Effective CFL $c_{\text {eff }}$, of SSP Linear $(m, p)$ Runge-Kutta Methods

\begin{tabular}{rlllllllllll}
\hline & $\mathrm{p}$ & 1 & 2 & 3 & 4 & 5 & 6 & 7 & 8 & 9 & 10 \\
$\mathrm{~m}$ & & & & & & & & & & & \\
\hline 1 & $c$ & 1 & & & & & & & & \\
2 & $c$ & 2 & 1 & & & & & & & \\
3 & $c$ & 3 & 2 & 1 & & & & & & & \\
4 & $c$ & 4 & 3 & 2 & 1 & & & & & & \\
5 & $c$ & 5 & 4 & 2.6506 & 2 & 1 & & & & & \\
6 & $c$ & 6 & 5 & 3.5184 & 2.6506 & 2 & 1 & & & & \\
7 & $c$ & 7 & 6 & 4.2879 & 3.5184 & 2.6506 & 2 & 1 & & & \\
8 & $c$ & 8 & 7 & 5.1071 & 4.2879 & 3.3733 & 2.6506 & 2 & 1 & & \\
9 & $c$ & 9 & 8 & 6 & 5.1071 & 4.1000 & 3.3733 & 2.6506 & 2 & 1 & \\
10 & $c$ & 10 & 9 & 6.7853 & 6 & 4.8308 & 4.1000 & 3.3733 & 2.6506 & 2 & \\
1 & $c_{\text {eff }}$ & 1 & & & & & & & & & \\
2 & $c_{\text {eff }}$ & 1 & 0.5 & & & & & & & & \\
3 & $c_{\text {eff }}$ & 1 & 0.6666 & 0.3333 & & & & & & & \\
4 & $c_{\text {eff }}$ & 1 & 0.75 & 0.5 & 0.25 & & & & & & \\
5 & $c_{\text {eff }}$ & 1 & 0.8 & 0.5301 & 0.4 & 0.2 & & & & & \\
6 & $c_{\text {eff }}$ & 1 & 0.8333 & 0.5864 & 0.4417 & 0.3333 & 0.1666 & & & & \\
7 & $c_{\text {eff }}$ & 1 & 0.8571 & 0.6125 & 0.5026 & 0.3786 & 0.2857 & 0.1428 & & & \\
8 & $c_{\text {eff }}$ & 1 & 0.875 & 0.6383 & 0.5359 & 0.4216 & 0.3313 & 0.25 & 0.125 & & \\
9 & $c_{\text {eff }}$ & 1 & 0.8888 & 0.6666 & 0.5674 & 0.4555 & 0.3748 & 0.2945 & 0.2222 & 0.1111 & \\
10 & $c_{\text {eff }}$ & 1 & 0.9 & 0.67853 & 0.6 & 0.48308 & 0.41000 & 0.33733 & 0.26506 & 0.2 & 0.1 \\
\hline
\end{tabular}

Table II. Coefficients $\alpha_{m, j}$ of SSP Linear (m, m), with CFL Coefficient $c=1$

\begin{tabular}{lllllllll}
\hline Order $m$ & $\alpha_{m, 0}$ & $\alpha_{m, 1}$ & $\alpha_{m, 2}$ & $\alpha_{m, 3}$ & $\alpha_{m, 4}$ & $\alpha_{m, 5}$ & $\alpha_{m, 6}$ & $\alpha_{m, 7}$ \\
\hline 1 & 1 & & & & & & & \\
2 & $\frac{1}{2}$ & $\frac{1}{2}$ & & & & & & \\
3 & $\frac{1}{3}$ & $\frac{1}{2}$ & $\frac{1}{6}$ & & & & & \\
4 & $\frac{3}{8}$ & $\frac{1}{3}$ & $\frac{1}{4}$ & $\frac{1}{24}$ & & & & \\
5 & $\frac{11}{30}$ & $\frac{3}{8}$ & $\frac{1}{6}$ & $\frac{1}{12}$ & $\frac{1}{120}$ & & & \\
6 & $\frac{53}{144}$ & $\frac{11}{30}$ & $\frac{3}{16}$ & $\frac{1}{18}$ & $\frac{1}{48}$ & $\frac{1}{720}$ & & \\
7 & $\frac{103}{280}$ & $\frac{53}{144}$ & $\frac{11}{60}$ & $\frac{3}{48}$ & $\frac{1}{72}$ & $\frac{1}{240}$ & $\frac{1}{5040}$ & \\
8 & $\frac{2119}{5760}$ & $\frac{103}{280}$ & $\frac{53}{288}$ & $\frac{11}{180}$ & $\frac{1}{64}$ & $\frac{1}{360}$ & $\frac{1}{1440}$ & $\frac{1}{40320}$ \\
\hline
\end{tabular}


SSPRK linear (m, 1): ([6] Proposition 2.2) The $m$ stage, first order SSP RK method given by

$$
\begin{aligned}
u^{(0)} & =u^{n}, \\
u^{(i)} & =\left(1+\frac{\Delta t}{m} L\right) u^{(i-1)}, \quad i=1, \ldots, m, \\
u^{n+1} & =u^{(m)}
\end{aligned}
$$

has CFL coefficient $c=m$, which is optimal among in the class of $m$ stage, order $p=1$ methods with nonnegative coefficients. This allows for a larger timestep but the computational cost increases correspondingly. This is reflected by the fact that the effective CFL is $c_{\text {eff }}=1$, which is equivalent to the forward Euler method.

SSPRK linear (m, 2): ([6] method 1) The $m$ stage, second order SSP methods:

$$
\begin{aligned}
u^{(0)} & =u^{n} \\
u^{(i)} & =\left(1+\frac{\Delta t}{m-1} L\right) u^{(i-1)}, \quad i=1, \ldots, m-1, \\
u^{m} & =\frac{1}{m} u^{(0)}+\frac{m-1}{m}\left(1+\frac{\Delta t}{m-1} L\right) u^{(m-1)}, \\
u^{n+1} & =u^{(m)} .
\end{aligned}
$$

have an optimal CFL coefficient $c=m-1$ among all methods with nonnegative coefficients. Although these methods were designed for linear problems, they methods are also nonlinearly second order [31]. Each such method uses $m$ stages to attain the order usually obtained by a 2-stage method, but has CFL coefficient $c=m-1$, thus the effective CFL coefficient here is $c_{\text {eff }}=m-1 / m$.

SSPRK linear (m, m-1): ([6] method 2) The $m$ stage, order $p=m-1$ method:

$$
\begin{aligned}
u^{(0)} & =u^{n}, \\
u^{(i)} & =u^{(i-1)}+\frac{1}{2} \Delta t L u^{(i-1)}, \quad i=1, \ldots, m-1, \\
u^{(m)} & =\sum_{k=0}^{m-2} \alpha_{m, k} u^{(k)}+\alpha_{m, m-1}\left(u^{(m-1)}+\frac{1}{2} \Delta t L u^{(m-1)}\right), \\
u^{n+1} & =u^{(m)},
\end{aligned}
$$


Table III. Coefficients $\alpha_{m, j}$ of SSP Linear (m, m-1), Which Have CFL Number $c=2$

\begin{tabular}{ccccccccccc}
\hline Stages $m$ & $\alpha_{m, 0}$ & $\alpha_{m, 1}$ & $\alpha_{m, 2}$ & $\alpha_{m, 3}$ & $\alpha_{m, 4}$ & $\alpha_{m, 5}$ & $\alpha_{m, 6}$ & $\alpha_{m, 7}$ & $\alpha_{m, 8}$ & $\alpha_{m, 9}$ \\
\hline 2 & 0 & 1 & & & & & & & & \\
3 & $\frac{1}{3}$ & 0 & $\frac{2}{3}$ & & & & & & & \\
4 & 0 & $\frac{2}{3}$ & 0 & $\frac{1}{3}$ & & & & & & \\
5 & $\frac{1}{5}$ & 0 & $\frac{2}{3}$ & 0 & $\frac{2}{15}$ & & & & & \\
6 & $\frac{1}{9}$ & $\frac{2}{5}$ & 0 & $\frac{4}{9}$ & 0 & $\frac{2}{45}$ & & & & \\
7 & $\frac{1}{7}$ & $\frac{2}{9}$ & $\frac{2}{5}$ & 0 & $\frac{2}{9}$ & 0 & $\frac{4}{315}$ & & & \\
8 & $\frac{2}{15}$ & $\frac{2}{7}$ & $\frac{2}{9}$ & $\frac{4}{15}$ & 0 & $\frac{4}{45}$ & 0 & $\frac{1}{315}$ & & \\
9 & $\frac{11}{81}$ & $\frac{4}{15}$ & $\frac{2}{7}$ & $\frac{4}{27}$ & $\frac{2}{15}$ & 0 & $\frac{4}{135}$ & 0 & $\frac{2}{2835}$ & \\
10 & $\frac{71}{525}$ & $\frac{22}{81}$ & $\frac{4}{15}$ & $\frac{4}{21}$ & $\frac{2}{27}$ & $\frac{4}{75}$ & 0 & $\frac{8}{945}$ & 0 & $\frac{2}{14175}$ \\
\hline
\end{tabular}

where the coefficients are given by:

$$
\begin{aligned}
\alpha_{2,0} & =0 \quad \alpha_{2,1}=1, \\
\alpha_{m, k} & =\frac{2}{k} \alpha_{m-1, k-1}, \quad k=1, \ldots, m-2, \\
\alpha_{m, m-1} & =\frac{2}{m} \alpha_{m-1, m-2}, \quad \alpha_{m, 0}=1-\sum_{k=1}^{m-1} \alpha_{m, k}
\end{aligned}
$$

is SSP with optimal (for methods with nonnegative coefficients) CFL coefficient $c=2$. Table III includes the coefficients of these methods up to order 10. The effective CFL for these methods is $c_{\text {eff }}=2 / \mathrm{m}$.

It is possible to extend these results to the case of a constant linear operator with a time dependent forcing term [6,30]. This is a case which arises in linear PDEs with time dependent boundary conditions such as Maxwell's equations which arise in computational electromagnetics (see [2]), and can be written as:

$$
u_{t}=L u+f(t)
$$

where $u=\left[u_{i}\right]$ is a vector, $L=\left[L_{i, j}\right]$ is a constant matrix and $f(t)=\left[f_{i}(t)\right]$ is a vector of functions of $t$. If the functions $f(t)$ can be written in a suitable way, then Eq. (2.8) can be converted to a linear constant-coefficient ODE. $f(t)$ is written, or approximated, as: 


$$
f_{i}(t)=\sum_{j=0}^{n} a_{j}^{i} q_{j}(t)=[A q(t)]_{i}
$$

where $A=\left[A_{i, j}\right]=\left[a_{j}^{i}\right]$ is a constant matrix and $q(t)=\left[q_{j}(t)\right]$ are a set of functions which have the property that $q^{\prime}(t)=D q(t)$, where $D$ is a constant matrix. Once the approximation to $f(t)$ is obtained, the ODE (2.8) can be converted into the linear, constant coefficient ODE

$$
y_{t}=M y(t)
$$

where

$$
y(t)=\left(\begin{array}{c}
q(t) \\
u(t)
\end{array}\right) \quad \text { and } \quad M=\left(\begin{array}{cc}
D & 0 \\
A & L
\end{array}\right)
$$

Thus, an equation of the form (2.8) can be approximated (or given exactly) by a linear constant coefficient ODE, and the SSP RK methods derived in this section can be applied.

\subsection{The Need for the SSP Property in the Intermediate Stages}

In practice, one of the major stability requirements on the RK method is that it be SSP for the internal stages. This means that it is not sufficient for $\left\|u^{n+1}\right\| \leqslant\left\|u^{n}\right\|$, but that each intermediate calculation $u^{(i)}$ for $i=1, \ldots, m$ must also satisfy $\left\|u^{(i)}\right\| \leqslant\left\|u^{(i-1)}\right\|$. This condition is frequently necessary in the approximation of the solution of hyperbolic PDEs. For example, in the numerical solution of the Euler equations of gas dynamics, it is imperative that negative pressure or density will be avoided even in the intermediate stages. This can be guaranteed if the intermediate stages are SSP. Since the proof of Theorem 2.1 relies on convexity arguments, which are satisfied at the intermediate stages as well, SSP RK methods have also intermediate stage SSP properties.

The following numerical example demonstrates how even for a simple, linear problem with a linear method, the SSP property is needed to ensure that the intermediate stage does not develop oscillations. Consider the differential equation $u_{t}-u_{x}=0$ on the domain $0 \leqslant x \leqslant 1$ with a step function initial condition

$$
u(0, x)= \begin{cases}0 & \text { if } x \leq \frac{1}{2} \\ 1 & \text { if } x>\frac{1}{2}\end{cases}
$$



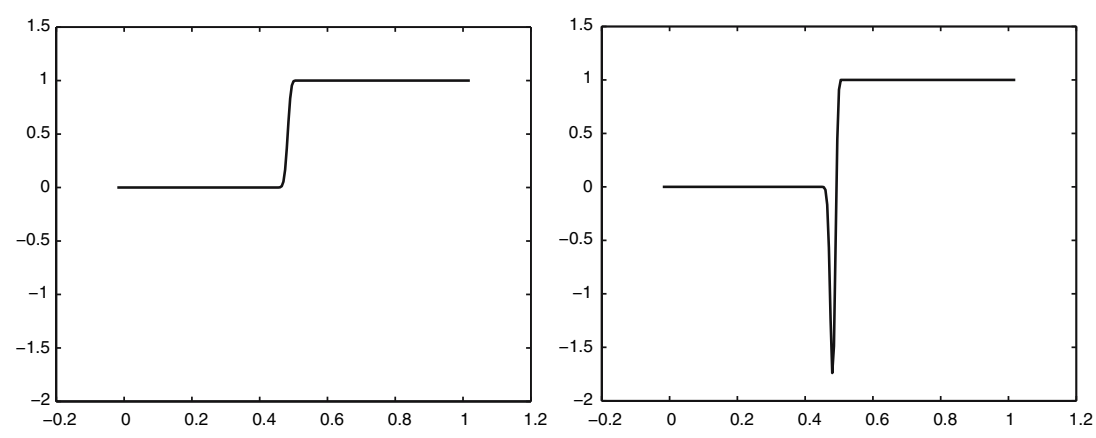

Fig. 1. First order one sided spatial discretization. Intermediate stage solution $u^{(1)}$ after 10 time steps. Left: SSP time discretization; right: nonSSP time discretization.

The spatial discretization is achieved by the first order one sided difference

$$
u_{t}=L(u)=\frac{u\left(t, x_{j+1}\right)-u\left(t, x_{j}\right)}{\Delta x},
$$

where the notation $x_{j}$ indicated the jth spatial grid point. Note that this spatial discretization is linear.

Figure 1 shows that using the second order SSP RK spatial discretization $\operatorname{SSPRK}(2,2)$, we obtain a solution with no osillations even in the intermediate stages. However, the second order nonSSP RK

$$
\begin{aligned}
& u^{(1)}=u^{n}-20 \Delta t L\left(u^{n}\right), \\
& u^{(2)}=u^{n}+\frac{41}{40} \Delta t L\left(u^{n}\right)-\frac{1}{40} \Delta t L\left(u^{(1)}\right)
\end{aligned}
$$

gives us a large undershoot at the intermediate stage.

It is clear, then, that the SSP guarantee of provable stability is necessary even for the intermediate stages, and is given with no additional cost by the methods constructed in this section.

\section{MULTI STEP METHODS}

Explicit SSP multi step methods:

$$
u^{n+1}=\sum_{i=1}^{m}\left(\alpha_{i} u^{n+1-i}+\Delta t \beta_{i} L\left(u^{n+1-i}\right)\right), \quad \alpha_{i} \geqslant 0 .
$$

are conveniently easy to manipulate into convex combinations of forward Euler steps. Just as in the Shu-Osher representation of RK schemes, since 
$\sum \alpha_{i}=1$, it follows that $u^{n+1}$ is given by a convex combination of forward Euler solvers, with suitably scaled $\Delta t$ 's, and so it becomes clear that the SSP property will apply to such multi step methods:

Theorem 3.1. [28]. If the forward Euler method combined with the spatial discretization $L$ in (2.3) is strongly stable under the CFL restriction (2.4), $\left\|u^{n}+\Delta t L\left(u^{n}\right)\right\| \leqslant\left\|u^{n}\right\|$, and if Euler's method solved backward in time in combination with the spatial discretization $\tilde{L}$ in (2.6) is also strongly stable under the CFL restriction (2.4), $\left\|u^{n}-\Delta t \tilde{L}\left(u^{n}\right)\right\| \leqslant\left\|u^{n}\right\|$, then the multi-step method (3.1) is SSP $\left\|u^{n+1}\right\| \leqslant\left\|u^{n}\right\|$, under the CFL restriction

$$
\Delta t \leqslant c \Delta t_{F E}, \quad c=\min _{i} \frac{\alpha_{i}}{\left|\beta_{i}\right|},
$$

provided $\beta_{i} L(\cdot)$ is replaced by $\beta_{i} \tilde{L}(\cdot)$ whenever $\beta_{i}$ is negative.

For SSP multi step schemes, it was shown ([8]) that for $m \geqslant 2$, there is no $m$ step, $m$-th order SSP method with all nonnegative $\beta_{i}$, and there is no $m$ step SSP method of order $(m+1)$. Thus, the ideas that came up in the context of SSPRK methods, namely increasing the number of stages and considering the downwind operator $\tilde{L}$ are both of relevance in the context of SSP multi step methods.

Table IV contains two optimal second order methods ([8] pp 105106), the two step method (scheme 1) and the three step method (scheme 2 ), both of which have $c=1 / 2$. Note that because of the negative $\beta$ in the scheme 1 , scheme 2 is actually more efficient. This is because at each time level, $L$ and $\tilde{L}$ have to be computed for scheme 1 , but only $L$ has to be computed for scheme 2. Although not provably optimal, scheme 3 (four step, second order) is still more efficient, with a CFL coefficient $c=2 / 3$, but only one computation per time step.

Of the third order methods (schemes 4-6 in Table IV) the four step method (scheme 4) is optimal with CFL coefficient $c=1 / 3$ ([8] p. 106). However, adding steps increases the CFL number, without requiring additional computation, only additional storage. Schemes 5 and 6 , though not proven optimal, increase the CFL to $c=1 / 2$ and $c=0.567$, respectively.

Unfortunately, the fourth (schemes 7-9) and fifth order (schemes 10-11) methods typically have very small CFL coefficients which preclude their use. These schemes are not proven optimal, either theoretically or numerically, but are the best ones found to date.

The restrictive CFL coefficients are the inevitable result of requiring the SSP property to hold for arbitrary starting values. An illustration of 
Table IV. Coefficients of SSP Multi Step Methods with Order $r$ and $m$ Steps. The Methods Marked with an * Were Proven Optimal in [8]

\begin{tabular}{llllll}
\hline$\#$ & $\begin{array}{c}\text { steps } \\
m\end{array}$ & order & CFL & \multicolumn{1}{l}{$\alpha_{i}$} & \multicolumn{1}{l}{$\beta_{i}$} \\
\hline $1^{*}$ & 2 & 2 & $\frac{1}{2}$ & $\frac{4}{5}, \frac{1}{5}$ & $\frac{8}{5},-\frac{2}{5}$ \\
$2^{*}$ & 3 & 2 & $\frac{1}{2}$ & $\frac{3}{4}, 0, \frac{1}{4}$ & $\frac{3}{2}, 0,0$ \\
3 & 4 & 2 & $\frac{2}{3}$ & $\frac{8}{9}, 0,0, \frac{1}{9}$ & $\frac{4}{3}, 0,0,0$ \\
4 & 4 & 3 & $\frac{1}{3}$ & $\frac{16}{27}, 0,0, \frac{11}{27}$ & $\frac{16}{9}, 0,0, \frac{4}{9}$ \\
5 & 5 & 3 & $\frac{1}{2}$ & $\frac{25}{32}, 0,0,0, \frac{7}{32}$ & $\frac{25}{16}, 0,0,0, \frac{5}{16}$ \\
$6 *$ & 6 & 3 & 0.567 & $\frac{108}{125}, 0,0,0,0, \frac{17}{125}$ & $\frac{36}{25}, 0,0,0,0, \frac{6}{25}$ \\
7 & 4 & 4 & 0.159 & $\frac{1989}{5000}, \frac{2893}{10000}, \frac{517}{2000}, \frac{34}{625}$ & $\frac{601613}{240000},-\frac{1167}{640}, \frac{130301}{80000},-\frac{82211}{240000}$ \\
8 & 6 & 4 & 0.245 & $\frac{747}{1280}, 0,0,0, \frac{81}{256}, \frac{1}{10}$ & $\frac{237}{128}, 0,0,0, \frac{165}{128},-\frac{3}{8}$ \\
9 & 5 & 4 & 0.021 & $\frac{1557}{32000}, \frac{1}{32000}, \frac{1}{120}, \frac{2063}{48000}, \frac{9}{10}$ & $\frac{5323561}{2304000}, \frac{2659}{2304000}, \frac{904987}{2304000}, \frac{1567579}{768000}, 0$ \\
10 & 5 & 5 & 0.085 & $\frac{1}{4}, \frac{13}{50}, \frac{8}{25}, \frac{7}{50}, \frac{3}{100}$ & $\frac{52031}{18000},-\frac{26617}{9000}, \frac{1412}{375},-\frac{14407}{9000}, \frac{6161}{18000}$ \\
11 & 6 & 5 & 0.130 & $\frac{7}{20}, \frac{3}{10}, \frac{4}{15}, 0, \frac{7}{120}, \frac{1}{40}$ & $\frac{291201}{108000},-\frac{198401}{86400}, \frac{88063}{43200}, 0,-\frac{17969}{43200}, \frac{73061}{432000}$ \\
\hline
\end{tabular}

the difficulty is given in [14]: Consider the simple example of the wellknown BDF2 method applied to the problem $u^{\prime}(t)=0$ :

$$
u_{2}=\frac{4}{3} u_{1}-\frac{1}{3} u_{0} .
$$

Clearly, this method is not SSP ( $\alpha_{2}$ is negative!). In other words, it is not always possible to obtain $\left\|u_{2}\right\| \leqslant\left\|u_{0}\right\|$ whenever $\left\|u_{1}\right\| \leqslant\left\|u_{0}\right\|$. However, it is also clear that the only relevant choice for this problem is $u_{1}=u_{0}$, and in this case we do obtain (trivially) $\left\|u_{2}\right\| \leqslant\left\|u_{0}\right\|$. Using this idea, Hundsdorfer, Ruuth, and Spiteri [14] examined the required stepsize for several multi step methods with particular starting procedures. These multi step methods do not satisfy the SSP conditions, however, with suitable starting procedures they, too, can be shown to be SSP. This creative approach to SSP multi step methods demonstrates that the SSP criteria may sometimes be relaxed or replaced by other conditions on the method.

\section{IMPLICIT SSP METHODS}

Implicit methods are desirable as they typically eliminate the step-size restriction associated with stability analysis. In [8] we presented examples 
of spatial discretizations which possess strong stability properties for implicit Euler. In fact, it was shown in $[12,14]$, that any spatial discretization $L$, which is strongly stable in some norm for the explicit forward Euler method under a certain time restriction will also be strongly stable, in the same norm, for the implicit Euler method, without a time restriction.

In [8], a compelling numerical example demonstrated that a nonSSP implicit method can destroy the nonoscillatory property of the implicitEuler method for a linear wave equation, despite the use of a nonoscillatory spatial discretization. The goal in this section is to present the efforts which have been made to design higher order implicit methods which share the strong stability properties of implicit-Euler, without any restriction on the time step $\Delta t$. Unfortunately, this goal cannot be realized. For both RK and multi step methods it has been proved that any higher order SSP method, even for linear constant coefficient problems, will have some time-step restriction.

\subsection{Diagonally Implicit Runge-Kutta Methods}

A diagonally implicit RK method for (2.1) can be written in the form

$$
\begin{aligned}
u^{(0)} & =u^{n}, \\
u^{(i)} & =\sum_{k=0}^{i-1} \alpha_{i, k} u^{(k)}+\Delta t \beta_{i} L\left(u^{(i)}\right), \quad \alpha_{i, k} \geqslant 0, \quad i=1, \ldots, m, \\
u^{n+1} & =u^{(m)} .
\end{aligned}
$$

This form has only a single implicit $L$ term for each stage and no explicit forward Euler terms. This is to avoid time step restrictions for strong stability properties of explicit schemes. However, since explicit $L$ terms are contained indirectly beginning at the second stage from $u$ of the previous stages, we do not lose generality in writing the schemes as the form in (4.1) except for the absence of the $L\left(u^{(0)}\right)$ terms in all stages.

The assumption that the first order explicit Euler discretization is strongly stable under some timestep restriction implies that the implicit Euler discretization

$$
u^{n+1}=u^{n}+\Delta t L\left(u^{n+1}\right)
$$

is unconditionally strongly stable, $\left\|u^{n+1}\right\| \leqslant\left\|u^{n}\right\|$ [14]. If so, then (4.1) would be unconditionally strongly stable under the same norm provided $\beta_{i}>0$ for all $i$. If $\beta_{i}$ becomes negative, (4.1) would still be unconditionally strongly stable (under the same norm) as long as $\beta_{i} L$ is replaced by 
$\beta_{i} \tilde{L}$ whenever the coefficient $\beta_{i}<0$, where $\tilde{L}$ approximates the same spatial derivative(s) as $L$, but is unconditionally strongly stable under implicit Euler, backward in time:

$$
u^{n+1}=u^{n}-\Delta t \tilde{L}\left(u^{n+1}\right) .
$$

As before, from the numerical standpoint the difference is the change of upwind direction.

In [8] it was shown that if (4.1) is at least second order accurate, then $\alpha_{i, k}$ cannot be all nonnegative. This statement holds even if $L$ is linear. This result rules out the existence of SSP implicit RK schemes (4.1) of order higher than one, even for the linear constant coefficient problem.

If the explicit Euler terms are included in (4.1), the methods obtained with nonnegative coefficients would be SSP Runge-Kutta methods, but only under restrictions on $\Delta t$ similar to explicit methods. It is unclear whether there exist such methods with a CFL coefficient large enough to offset the cost of solving the implicit problem to obtain $u^{(i)}$-to date none have been found. This is an open area, and implicit RK methods with large CFL coefficient $(c \approx 10)$ would be of great interest.

\subsection{Implicit Multi-Step Methods}

Implicit SSP multi step methods can be written in the form

$$
u^{n+1}=\sum_{i=1}^{m} \alpha_{i} u^{n+1-i}+\Delta t \beta_{0} L\left(u^{n+1}\right), \quad \alpha_{i} \geqslant 0,
$$

which would be unconditionally SSP provided that $\beta_{0}>0$. If $\beta_{0}$ is negative, (4.2) would still be unconditionally strongly stable under the same norm if $L$ is replaced by $\tilde{L}$. Notice that (just as in the case of implicit RK methods) we have only a single implicit $L$ term and no explicit $L$ terms. This is to avoid time step restrictions for norm properties of explicit schemes. If explicit $L$ terms are included, we would be able to obtain SSP multi step methods under restrictions on $\Delta t$ similar to explicit methods. Unfortunately, there are no SSP implicit multi step schemes (4.2) of order higher than one [8]. This being the case, it makes sense to consider implicit $m$-step methods of the more general form:

$$
u^{n+1}=\sum_{i=1}^{m} \alpha_{i} u^{n+1-i}+\Delta t \sum_{i=0}^{m} \beta_{i} L\left(u^{n+1-i}\right), \quad \alpha_{i} \geqslant 0 .
$$

Although the inclusion of the explicit $L$ terms implies that this method can only be SSP with a stepsize restriction, the hope is that this 
stepsize restriction will not be severe, and that the larger stepsize will compensate for the additional computational work in solving the implicit problem.

In [14], Hundsdorfer, Ruuth and Spiteri explain that it follows from Lenferink's results on contractivity for linear systems [19] that, in general, any two step method of order $p>1$ would have CFL coefficient no greater than $c=2$. Of course, this provides a bound on the results for nonlinear problems as well. It is interesting to note that this bound is actually obtained, for example, by the Crank-Nicholson method. This second order method requires only one implicit computation and has CFL $c=2$ while the explicit RK method $\operatorname{SSPRK}(2,2)$ requires only two, with a CFL of $c=1$. Thus, $\operatorname{SSP}(2,2)$ requires four explicit computations while CrankNicholson requires one implicit computation for the same time step. However, the cost of solving the implicit problem is normally not offset by the cost of four explicit computations. Clearly, then, the bound of $c=2$ for second order implicit multi step methods is quite restrictive, and indicates that the use of implicit schemes is not computationally efficient.

Encouraged by their results in explicit multi step methods with suitable starting procedures, Hundsdorfer, Ruuth and Spiteri considered the case of implicit two step methods with different starting procedures (such as implicit Euler). However, their results show that even with suitable starting procedures, the stepsize restrictions for the implicit multi step methods are hardly better than those of explicit methods. Thus, implicit SSP multi step methods feature stepsize restrictions that are too severe to make the use of these methods feasible.

\section{MONOTONICITY AND CONTRACTIVITY RESULTS APPLIED TO SSP METHODS}

The class of SSP methods, which are based on the idea of decomposing time discretizations into convex combinations of forward Euler steps, was created by the hyperbolic PDE community to fill the need for stability criteria that did not rely on the linearity of the underlying problem or impose a smoothness assumption on the solution. It was a response to the fact that established RK theory did not provide a way to guarantee that the stability of Euler's method, when applied to an ODE resulting from a spatial discretization of a nonlinear discontinuous problem, will be preserved by a higher order time discretization method. An important and interesting shift has occured over the last few years, as experts in the field of RK methods have examined the class of SSP methods and the CFL number associated with each method and found this theory to be well connected to a large wealth of knowledge in established RK theory. This new 
connection, which will allow for future development and analysis of new SSP time discretizations, is described in this section.

Recently, several important papers by Ferracina and Spijker ([4, 5]) and Higueras $([12,13])$ have established the connection between the theory of absolute monotonicity and SSP, and provided practical methods to determine optimal schemes. These paper use Kraaijevanger's $([16,17])$ theory, which gave optimal step size restrictions for contractivity in terms of the radius of absolute stability, and explores order barriers for nonnegative radius of absolute monotonicity.

The RK methods are most commonly written not in the representation used above (2.5) but in the Butcher form

$$
\begin{aligned}
u^{(i)} & =u^{n}+\Delta t \sum_{j=1}^{m} a_{i j} L\left(u^{(j)}\right) \quad(1 \leqslant i \leqslant m), \\
u^{n+1} & =u^{n}+\Delta t \sum_{j=1}^{m} b_{j} L\left(u^{(j)}\right) .
\end{aligned}
$$

The notation $A=\left(a_{i j}\right)$ and $b=\left(b_{j}\right)$, allows any RK method given in the Butcher form to be referred to as $(A, b)$. Notice that this method may be fully implicit. Correspondingly, we can generalize the Shu-Osher representation to include the implicit terms, and to allow an easy conversion between the Shu-Osher representation and the Butcher representation [4]:

$$
\begin{aligned}
u^{(i)} & =\left(1-\sum_{j=1}^{m} \lambda_{i j}\right) u^{n}+\sum_{j=1}^{m}\left(\lambda_{i j} u^{(j)}+\Delta t \mu_{i j} L\left(u^{(j)}\right)\right) \quad(1 \leqslant i \leqslant m) \\
u^{n+1} & =\left(1-\sum_{j=1}^{m} \lambda_{m+1, j}\right) u^{n}+\sum_{j=1}^{m}\left(\lambda_{m+1, j} u^{(j)}+\Delta t \mu_{m+1, j} L\left(u^{(j)}\right)\right) .
\end{aligned}
$$

Clearly, then, if

$$
\mathcal{L}=\left(\begin{array}{c}
\mathcal{L}_{0} \\
\mathcal{L}_{1}
\end{array}\right)
$$

where $\mathcal{L}_{0}=\left(\lambda_{i j}\right)$ for $1 \leqslant i, j \leqslant m$, and $\mathcal{L}_{1}=\left(\lambda_{m+1, j}\right)$ for $1 \leqslant j \leqslant m$, and

$$
\mathcal{M}=\left(\begin{array}{c}
\mathcal{M}_{0} \\
\mathcal{M}_{1}
\end{array}\right)
$$

where $\mathcal{M}_{0}=\left(\mu_{i j}\right)$ for $1 \leqslant i, j \leqslant m$, and $\mathcal{M}_{1}=\left(\mu_{m+1, j}\right)$ for $1 \leq j \leq m$. 
To convert between the Shu-Osher representation and the Butcher array, we use

$$
\mathcal{M}_{0}=A-\mathcal{L}_{0} A \quad \mathcal{M}_{1}=b^{T}-\mathcal{L}_{1} A,
$$

(where $I-\mathcal{L}_{0}$ is invertible). Note that although any Shu-Osher representation will have a unique Butcher representation, the Butcher representation may have many Shu-Osher representations.

In the following, we deal only with irreducible RK methods - these are $m$ stage RK methods which are not equivalent, nor do they reduce to, methods which are of fewer stages. Given an irreducible RK scheme written in the Butcher array form, the radius of absolute monotonicity $R(A, b)$ of a method $(A, b)$ can be shown to be the optimal CFL coefficient $c$ for all SSPRK schemes $(\alpha, \beta)$ equivalent to $(A, b)[5,12,13]$. Furthermore, there is an explicit construction of an SSPRK scheme with such an optimal CFL coefficient $[5,13]$.

First, we need Kraaijevanger's conditions

$$
\begin{aligned}
I-\xi A & \text { is invertible } \\
A(I-\xi A)^{-1} & \geqslant 0, \\
(I-\xi A)^{-T} b & \geqslant 0, \\
(I-\xi A)^{-1} e & \geqslant 0, \\
1+\xi b^{T}(I-\xi A)^{-1} e & \geqslant 0,
\end{aligned}
$$

where ${ }^{-T}$ means the transpose of the inverse, and $e$ is the column vector whose components are all equal to 1 .

Definition. For a RK method written in Butcher form, if all the components of $A$ and $b$ are nonnegative, i.e. $A \geqslant 0$ and $b \geqslant 0$, the radius of absolute monotonicity, $R(A, b)$ is defined as

$R(A, b)=\sup \{r: r \geqslant 0$ and conditions 5.3 hold for all $\xi$ with $-r \leqslant \xi \leqslant 0\}$.

If $A \geqslant 0$ or $b \geqslant 0$ are violated, we define $R(A, b)=0$.

Based on Kraaijevanger's ([17]) theory, an algorithm to calculate $R(A, b)$ for any explicit RK method (A, b) is given in [4]. The connection between $R(A, b)$ and the CFL coefficient $c$, as well as the construction of the optimal Shu-Osher representation is given by $[4,5,12,13]$, and presented in the following theorem.

Theorem 5.2. ([5] Sec. 3 and [13] Sec. 2). Given a RK method defined by an irreducible coefficient scheme in Butcher form $(A, b)$ with radius of absolute monotonicity $R(A, b)>0$, any equivalent SSP RK 
method (in Shu-Osher representation) will be have CFL coefficient $c \leqslant$ $R(A, b)$. Moreover, a SSP RK method $(\mathcal{L}, \mathcal{M})$ can be obtained with CFL coefficient $c(\mathcal{L}, \mathcal{M})=R(A, b)$, (for $0 \leqslant R(A, b)<\infty)$, by the choice $\mathcal{L}$ :

$$
\begin{aligned}
\mathcal{L}_{0} & =\gamma A(I+\gamma A)^{-1}, \\
\mathcal{L}_{1} & =\gamma b^{T}(I+\gamma A)^{-1}, \\
\gamma & =R(A, b)
\end{aligned}
$$

and if $R(A, b)=\infty$ we use:

$$
\begin{aligned}
\mathcal{L}_{0} & =I-\gamma P \\
\mathcal{L}_{1} & =b^{T} P \\
\gamma & =\left(\max _{i} p_{i i}\right)^{-1}, \quad \text { where } P=\left(p_{i j}\right)=A^{-1} .
\end{aligned}
$$

As we see here, if a method $(A, b)$ with nonnegative $A$ and $b$ has radius of absolute monotonicity $R(A, b) \geqslant 0$, we can easily find the optimal ShuOsher representation for an SSP method with CFL coefficient $c=R(A, b)$. Given an RK method in the form $(A, b)$ with $A \geqslant 0$ and $b \geqslant 0$ with optimal $R(A, b)$ among all methods in its class, this theorem can be used to obtain the Shu-Osher representation of the optimal SSPRK method.

However, many methods do not have nonnegative $A$ and $b$ and some methods that do, have also $R(A, b)=0$. In such cases, the theorem above does not allow us to construct optimal methods. However, Higueras ([13] Sec. 3) has extended this theory to include the case where some elements of $A$ or $b$ may be negative, by considering perturbed RK methods. This is equivalent, in the Shu-Osher representation, to considering $\tilde{L}$. These results allow the extension of monotonicity and contractivity theory for RK methods to the theory of SSP RK methods.

\section{ACKNOWLEDGMENT}

Supported by NSF grant number DMS 0106743.

\section{REFERENCES}

1. Carpenter, M., and Kennedy, C. (1994). Fourth-order $2 N$-storage Runge-Kutta schemes, NASA TM 109112, NASA Langley Research Center.

2. Chen, M.-H., Cockburn, B., and Reitich, F. High order RKDG methods for computational electromagnetics. Submitted.

3. Cockburn, B., and Shu, C.-W. (1989). TVB Runge-Kutta local projection discontinuous Galerkin finite element method for conservation laws II: general framework, Math. Comput. 52, 411-435. 
4. Ferracina, L., and Spijker, M.N. (2002). Stepsize Restrictions for the total variation diminishing property in general Runge-Kutta methods. Num. Anal. Reports of Leiden University, Report MI 2002-21.

5. Ferracina, L., and Spijker, M.N. (2005). An extension and analysis of the Shu-Osher representation of Runge-Kutta method. Math. Comput. 74, 201-219.

6. Gottlieb, S., and Gottlieb, L.-J. (2003). Strong stability preserving properties of Runge-Kutta time discretization methods for linear constant coefficient operators, J. Sci. Compu. 18, $83-110$.

7. Gottlieb, S., and Shu, C.-W. (1998). Total variation diminishing Runge-Kutta schemes, Math. Сотрu. 67, 73-85.

8. Gottlieb, S., Shu, C.-W., and Tadmor, E. (2001). Strong stability preserving high-order time discretization methods, SIAM Review 43, 89-112.

9. Gottlieb, D., and Tadmor, E. (1995). The CFL condition for spectral approximations to hyperbolic initial-boundary value problems, Math. Comput. 56, 565-588.

10. Gottlieb, S., and Ruuth, S.J. Strong stability preserving Runge-Kutta methods for fast downwind biased discretizations, to appear in J. Sci. Comput.

11. Harten, A. (1983). High resolution schemes for hyperbolic conservation laws, J. Comput. Phys. 49, 357-393.

12. Higueras, I. (2004) On strong stability preserving methods. J. Sci Comput. 21,193-223.

13. Higueras, I. (2003). Representations of Runge-Kutta Methods and Strong Stability Preserving Methods, Preprint Departamento de Matematica e Informatica, No. 2, seccion 1, Universidad Publica de Navarra.

14. Hundsdorfer, W., Ruuth, S.J., and Spiteri, R.J. (2003). Monotonicity-preserving linear multistep methods. SIAM J. Num. Anal. 41, 605-623.

15. Kennedy, C., Carpenter, M., and Lewis, R. (2000). Low storage explicit Runge-Kutta schemes for the compressible navier-stokes equations, Appl. Nume. Math. 35, 177-219.

16. Kraaijevanger, J.F.B.M. (1986). Absolute monotonicity of polynomials occurring in the numerical solution of initial value problems, Numerische Mathematik 48, 303-322.

17. Kraaijevanger, J.F.B.M. (1991). Contractivity of Runge-Kutta methods, BIT 31, 482-528.

18. Kurganov, A., and Tadmor, E. New high-resolution schemes for nonlinear conservation laws and related convection-diffusion equations, UCLA CAM Report No. 99-16.

19. Lenferink, H.W.J. (1991). Contractivity preserving implicit linear multi step methods, Math. Comput. 56, 177-199.

20. Levy, D. and Tadmor, E. (1998). From semi-discrete to fully discrete: stability of RungeKutta schemes by the energy method. SIAM Review, 40, 40-73.

21. Liu, X-D., Osher, S., and Chan, T. (1994). Weighted essentially non-oscillatory schemes J. Comput. Phys. 115 (1), 200.

22. Nessyahu, H., and Tadmor, E. (1990). Non-oscillatory central differencing for hyperbolic conservation laws, J. Comp. Phys. 87, 408-463.

23. Osher, S., and Chakravarthy, S. (1984). High resolution schemes and the entropy condition. SIAM J. Num. Anal. 21, 955-984.

24. Osher, S., and Tadmor, E. (1988).On the convergence of difference approximations to scalar conservation laws. Math. Comp. 50, 19-51.

25. Ruuth, S.J., and Spiteri, R.J. (2002). Two barriers on strong-stability-preserving time discretization methods. J. Sci. Comp. 17, 211-220.

26. Ruuth, S.J., and Spiteri, R.J. (2004). Downwinding in high-order strong-stability-preserving Runge-Kutta methods. SIAM J. Numer. Anal. 42, 974-996.

27. Ruuth, S. Global optimization of strong-stability preserving Runge-Kutta methods, to appear in Math. Comput. 
28. Shu, C.-W. (1988). Total-variation-diminishing time discretizations. SIAM J. Sci. Stat. Comput. 9, 1073-1084.

29. Shu, C.-W., and Osher, S. (1998). Efficient implementation of essentially non-oscillatory shock-capturing schemes. J. Comput. Phy. 77, 439-471.

30. Shu, C.-W. (2002). A survey of strong stability preserving high order time discretizations. In Estep, D. and Tavener, S. Collected Lectures on the Preservation of Stability under Discretization SIAM, pp. 51-65.

31. Spiteri, R.J., and Ruuth, S.J., (2002). A new class of optimal high-order strong-stabilitypreserving time discretization methods. SIAM J. Numer. Anal. 40, 469-491.

32. Spiteri, R.J., and Ruuth, S.J. (2003). Nonlinear evolution using optimal fourth-order strong-stability-preserving Runge-Kutta methods. J. Math. Comput. Simul. 62, 125-135.

33. Strang, G. (1964). Accurate partial difference methods II: nonlinear problems. Numerische Mathematik 6, 37.

34. Strikwerda, J.C. Finite difference schemes and partial differential equations. Wadsworth and Brooks/Cole Mathematics Series. California 1989.

35. Sweby, P.K. (1984) High resolution schemes using flux limiters for hyperbolic conservation laws. SIAM J. Num. Anal. 21, 995-1011.

36. Tadmor, E. (1988). Approximate solutions of nonlinear conservation laws. In Quarteroni, A. (ed.), "Advanced Numerical Approximation of Nonlinear Hyperbolic Equations," Lectures Notes from CIME Course Cetraro, Italy, 1997 Lecture Notes in Mathematics 1697, Springer-Verlag, Berlin pp. 1-150.

37. Williamson, J.H. (1980). Low-storage Runge-Kutta schemes. J. Comput. Phy. 35, 48-56. 